\title{
Effects of Delayed-Release Olive Oil and Hydrolyzed Pine Nut Oil on Glucose Tolerance, Incretin Secretion and Appetite in Humans
}

\author{
Karina V. Sørensen ${ }^{1,2}$, Mads H. Kaspersen ${ }^{3}$, Jeppe H. Ekberg ${ }^{4,5}$, Annette Bauer-Brandl ${ }^{3}{ }^{\circledR}$, Trond Ulven ${ }^{3,4}{ }^{\circledR}$ \\ and Kurt Højlund 1,2,*(D) \\ 1 Steno Diabetes Center Odense, Odense University Hospital, 5000 Odense, Denmark; \\ karina.vejrum.sorensen@rsyd.dk \\ 2 Department of Clinical Research, University of Southern Denmark, 5000 Odense, Denmark \\ 3 Department of Physics, Chemistry and Pharmacy, University of Southern Denmark, 5230 Odense, Denmark; \\ mmk@sdu.dk (M.H.K.); annette.bauer@sdu.dk (A.B.-B.); tu@sund.ku.dk (T.U.) \\ 4 Department of Drug Design and Pharmacology, University of Copenhagen, 2100 Copenhagen, Denmark; \\ jpe@sund.ku.dk \\ 5 Center for Basic Metabolic Research, University of Copenhagen, 2200 Copenhagen, Denmark \\ * Correspondence: kurt.hoejlund@rsyd.dk; Tel.: +45-25320648
}

check for updates

Citation: Sørensen, K.V.; Kaspersen, M.H.; Ekberg, J.H.; Bauer-Brandl, A.; Ulven, T.; Højlund, K. Effects of Delayed-Release Olive Oil and Hydrolyzed Pine Nut Oil on Glucose Tolerance, Incretin Secretion and Appetite in Humans. Nutrients 2021, 13, 3407. https://doi.org/10.3390/ nu13103407

Received: 24 August 2021

Accepted: 23 September 2021

Published: 27 September 2021

Publisher's Note: MDPI stays neutral with regard to jurisdictional claims in published maps and institutional affiliations.

Copyright: (C) 2021 by the authors Licensee MDPI, Basel, Switzerland. This article is an open access article distributed under the terms and conditions of the Creative Commons Attribution (CC BY) license (https:// creativecommons.org/licenses/by/ $4.0 /)$.

\begin{abstract}
Background: To investigate the potential synergistic effects of olive oil releasing 2-oleoylglycerol and hydrolyzed pine nut oil containing $20 \%$ pinolenic acid on GLP-1 secretion, glucose tolerance, insulin secretion and appetite in healthy individuals, when delivered to the small intestine as potential agonists of GPR119, FFA1 and FFA4. Methods: Nine overweight/obese individuals completed three 6-h oral glucose tolerance tests (OGTTs) in a crossover design. At -30 min, participants consumed either: no oil, $6 \mathrm{~g}$ of hydrolyzed pine nut oil (PNO-FFA), or a combination of $3 \mathrm{~g}$ hydrolyzed pine nut oil and $3 \mathrm{~g}$ olive oil (PNO-OO) in delayed-release capsules. Repeated measures of glucose, insulin, C-peptide, GLP-1, GIP, ghrelin, subjective appetite and gastrointestinal tolerability were done. Results: PNO-FFA augmented GLP-1 secretion from 0-360 min compared to no oil and PNO-OO $(p<0.01)$. GIP secretion was increased from 240-360 min after both PNO-FFA and PNO-OO versus no oil $(p<0.01)$. Both oil treatments suppressed subjective appetite by reducing hunger and prospective food consumption and increasing satiety $(p<0.05)$. Conclusions: In support of previous findings, $6 \mathrm{~g}$ of delayed-release hydrolyzed pine nut oil enhanced postprandial GLP-1 secretion and reduced appetite. However, no synergistic effect of combining hydrolyzed pine nut oil and olive oil on GLP-1 secretion was observed. These results need further evaluation in long-term studies including effects on bodyweight and insulin sensitivity.
\end{abstract}

Keywords: G-protein-coupled receptors; pine nut oil; pinolenic acid; olive oil; 2-oleoylglycerol; incretins; glucose tolerance; appetite

\section{Introduction}

The free fatty acid receptor-1 (FFA1), free fatty acid receptor-4 (FFA4) and G-proteincoupled receptor-119 (GPR119) are G-protein-coupled receptors (GPCRs) activated by certain lipid metabolites. These receptors have caught the interest of researchers due to their modulatory effects on important metabolic responses including pancreatic insulin secretion, gastrointestinal secretion of incretin hormones, appetite regulation, insulin sensitization and anti-inflammation, effects with beneficial prospects for metabolic diseases such as obesity and type 2 diabetes.

FFA1 and FFA4 are activated by medium- and long-chain fatty acids [1], whereas GPR119 is known to respond to various lipid ligands, including 2-monoacylglycerols (2MAGs) [2,3]. FFA1, FFA4 and GPR119 are expressed in the gastrointestinal tract in enteroendocrine cells (EC) [2,4-6]. Activation of the receptors in ECs has been shown to stimulate the secretion of incretins such as glucagon-like peptide-1 (GLP-1) and glucosedependent insulinotropic peptide (GIP) [2,4-6], although this effect has been questioned for 
FFA4 [7]. The incretin hormones are capable of enhancing glucose-stimulated insulin secretion (GSIS) [8]. Furthermore, GLP-1 has additional effects including appetite suppression leading to subsequent loss of body weight when used in pharmacological doses $[8,9]$. FFA1 and GPR119 are also present on pancreatic beta-cells where they enhance GSIS $[10,11]$. The potential of stimulating FFA1 was convincingly demonstrated in a phase 3 trial, where the pharmaceutical FFA1 agonist TAK-875 was able to reduce glycated hemoglobin levels in patients with type 2 diabetes. However, further studies of this drug were terminated due to liver toxic side effects [12]. FFA4 is also present on ghrelin secreting cells, and it has been demonstrated that FFA4 activation inhibits ghrelin secretion in humans [13]. Ghrelin is an orexigenic hormone playing a central role in appetite control [14]. Thus, a reduction in ghrelin levels can potentially reduce appetite and decrease energy intake and body weight. Moreover, in animal and cell studies, the activation of FFA4 on macrophages and in adipose tissue has been reported to reduce inflammation and improve insulin sensitivity $[15,16]$.

In a recent study, screening of 46 fatty acids demonstrated pinolenic acid to be the most potent dual agonist of human FFA1 and FFA4 in vitro [1]. Moreover, pinolenic acid and Siberian pine nut oil (containing $20 \%$ pinolenic acid) were shown to reduce glucose levels compared to maize oil during an oral glucose tolerance test (OGTT) in mice, with a superior effect of pinolenic acid [1]. We further evaluated these effects in a clinical study testing the effects of delayed-release hydrolyzed pine nut oil versus genuine unhydrolyzed pine nut oil prior to an OGTT [17]. While hydrolyzed pine nut oil caused only a small reduction in glucose levels from $0-120 \mathrm{~min}$, we observed a more pronounced increase in circulating GLP-1 levels and a concomitant reduction in ghrelin levels from 120-240 min compared with no oil control treatment. Moreover, subjective appetite was reduced. Thus, hydrolyzed pine nut oil delivered to the small intestine by delayed-release capsules may be a beneficial functional dietary lipid able to stimulate GLP-1 secretion and reduce ghrelin levels late in the postprandial state causing an appetite reducing effect.

GPR119 in transfected cell lines can be activated by a number of 2MAGs including 2-oleoylglycerol (2OG) [3], comprising an oleic acid attached to the sn-2 position of glycerol. Though oleoylethanolamide is a more potent endogenous agonist of GPR119, the much higher abundancy of $2 \mathrm{OG}$ (and other 2MAGs) in the intestine, formed as metabolic product during digestion and absorption of triglycerides, outweighs the lower potency of 2OG and makes it a better dietary candidate for activating GPR119 [3]. Purified 2OG (2 g) administered through a nasojejunal tube and also ordinary oral intake of olive oil ( $19 \mathrm{~g}$, liberating $7.7 \mathrm{~g} 2 \mathrm{OG}$ upon complete digestion) have been shown to effectively stimulate GLP-1 release in humans [18-20]. Interestingly, it was recently demonstrated, using specific tool compounds in a cell model, that simultaneous activation of GPR119 and FFA1 act in synergy to enhance enteroendocrine GLP-1 secretion beyond the combined individual responses [7].

The aim of this study was to evaluate the potential synergistic effect of combined activation of FFA1, FFA4 and GPR119 by intake of delayed-release hydrolyzed pine nut oil (containing pinolenic acid) and olive oil (liberating 2OG upon digestion) on incretin hormones, glucose tolerance, insulin secretion, insulin sensitivity and appetite in vivo in healthy humans.

\section{Materials and Methods}

The study was performed at Steno Diabetes Center Odense, Odense University Hospital, Denmark, and was approved by the Regional Committees on Health Research Ethics for Southern Denmark (S-20150060). Moreover, it was conducted in accordance with the Helsinki Declaration.

\subsection{Participants}

Participants were included in the study based on the following criteria: age from 40-70 years, BMI 27.5-40 kg $/ \mathrm{m}^{2}$, normal glucose tolerance (2-h plasma glucose $<7.8 \mathrm{mmol} / \mathrm{L}$ ), normal screening blood samples (within Danish reference ranges) including markers of 
kidney and liver function, cholesterols, triglycerides and hematology. Participants had to be healthy with a normal blood pressure $(140 / 90 \mathrm{mmHg})$, no need for prescriptive medicine, no chronic diseases including gastrointestinal diseases or previous gastrointestinal surgery, no first-degree relatives with diabetes, and no food allergies of importance. Moreover, they had to be non-smokers with a stable body weight $(<3 \mathrm{~kg}$ change within three months prior) and no intake of dietary supplements (within 1 month prior) or adherence to any type of restrictive diet (calorie restriction, vegan diet, etc.). All participants gave written informed consent prior to any study related procedures.

Out of eleven pre-screened participants invited to the initial screening OGTT, two were excluded due to impaired glucose tolerance. Thus, nine participants completed the study protocol. Baseline characteristics are shown in Table 1.

Table 1. Baseline characteristics $(n=9)$.

\begin{tabular}{cc}
\hline Age (Years) & $54 \pm 8$ \\
Sex, m/f $(n)$ & $4 / 5$ \\
Height (cm) & $174 \pm 9$ \\
Weight (kg) & $105 \pm 21$ \\
BMI (kg/m²) & $34.4 \pm 4.2$ \\
Fat mass (kg) & $42 \pm 10$ \\
Fat free mass (kg) & $61 \pm 17$ \\
Fat \% & $41 \pm 8$ \\
Systolic blood pressure (mmHg) & $135 \pm 13$ \\
Diastolic blood pressure (mmHg) & $85 \pm 5$ \\
Plasma glucose (mmol/L) & $5.3 \pm 0.4$ \\
Creatinine (umol/L) & $77 \pm 10$ \\
Triglycerides (mmol/L) & $1.2 \pm 0.3$ \\
HDL-cholesterol (mmol/L) & $1.5 \pm 0.4$ \\
LDL-cholesterol (mmol/L) & $3.1 \pm 0.5$ \\
Total cholesterol (mmol/L) & $5.1 \pm 0.6$ \\
HOMA-IR * & $3.0 \pm 1.4$ \\
\hline
\end{tabular}

* Homeostatic model assessment of insulin resistance (HOMA-IR) calculated as: fasting glucose mmol/L $\times$ fasting insulin $\mathrm{mU} / \mathrm{L} / 22.5$. Values are mean $\pm \mathrm{SD}$.

\subsection{Study Design}

The study was a crossover trial with three different arms. The first arm was a 6-h OGTT (75 g glucose) with no oil intake prior to the test corresponding to the initial screening OGTT for the nine participants. Hereafter, two additional OGTTs were completed in a randomized order (www.randomizer.org, 2 May 2018) consuming either $6 \mathrm{~g}$ of hydrolyzed pine nut oil (PNO-FFA) alone or $3 \mathrm{~g}$ hydrolyzed pine nut oil combined with $3 \mathrm{~g}$ olive oil (PNO-OO), in both cases together with $160 \mathrm{~mL}$ of water $30 \mathrm{~min}$ prior to the OGTT. The same amount of water was also consumed $30 \mathrm{~min}$ prior to the screening/control OGTT. A washout period of one to four weeks between the OGTTs was applied (Supplementary Figure S1). During all OGTTs sampling of blood was done at $-30,-15$ and $0 \mathrm{~min}$, and every half hour until $240 \mathrm{~min}$, and additionally at 300 and $360 \mathrm{~min}$. Prior to all experimental days, participants consumed a self-made standard evening meal with no calorie restriction and were instructed to fast from 10 PM after intake of the meal (a small amount of water was allowed). Furthermore, participants were instructed to abstain from physical exercise and alcohol consumption $48 \mathrm{~h}$ prior to the experimental days and to attend the research facility by car or a non-strenuous way of transportation. All participants were instructed to maintain their habitual lifestyle until the end of the study. The study was performed from June to December 2018.

\subsection{Subjective Appetite and Gastrointestinal Tolerability}

Appetite was assessed by the use of visual analog scale (VAS) questionnaires including hunger, satiety, fullness, prospective food consumption and overall well-being [21]. The degree of each symptom was indicated on a $100 \mathrm{~mm}$ horizontal line, where 0 and $100 \mathrm{~mm}$ 
indicated the extreme feelings related to the symptom in question. At every OGTT appetite questionnaires were completed at $-30,-15,0,15,45,60 \mathrm{~min}$ and every half hour until $240 \mathrm{~min}$ and hereafter at 300 and $360 \mathrm{~min}$.

Similarly, we used VAS questionnaires to measure gastrointestinal tolerability by assessing the following symptoms: nausea/vomiting, bloating, flatulence, constipation, diarrhea and abdominal pain [22]. Questions were formulated to reflect the acute setting of the test. Again, $0 \mathrm{~mm}$ and $100 \mathrm{~mm}$ indicated the extreme degree of the symptom in question, that is: no degree of the symptom or a severe degree. This was done prior to the OGTT, after the OGTT and at 8:00 p.m. on the same evening and 8:00 a.m. the morning after.

\subsection{Indices of Insulin Sensitivity and Beta-Cell Function}

OGTT based insulin sensitivity was determined by BIGTT Si $_{1}(0,30$ and $120 \mathrm{~min})$ [23] and MATSUDA $(0,30,60,90$ and $120 \mathrm{~min})$ [24], and beta-cell function was determined by $\operatorname{BIGTT}_{\text {AIR }}(0,30$ and $120 \mathrm{~min})$ [23] and $\mathrm{CIR}_{30 \mathrm{~min}}$ [25].

\subsection{Analysis of Blood Biomarkers}

Plasma glucose was analyzed by an ABL800 FLEX blood gas analyzer, and serum insulin and C-peptide were analyzed on a Cobas e 411 with an electrochemiluminescence immunoassay (ECLIA). Insulin intra- and inter-assays had a CV\% of 1.9-2.0 and 2.5-2.6, respectively. The C-peptide intra- and inter-assay CV\% were 1.3-4.6 and 1.8-5.0, respectively. Plasma free fatty acids (FFA) were analyzed using the in vitro enzymatic colorimetric method assay NEFA-HR(2) (Wako Chemicals, GmbH, Neuss, Germany). Plasma GLP-1 was assessed by the Total GLP-1 (ver. 2) Assay Kit from Meso Scale Discovery. Plasma GIP and ghrelin were assessed by using the Human GIP (Total) or Human Ghrelin (Total) ELISA Kit from Millipore. Intra- and inter-assay CV\% of the GIP kit was 3.0-8.8 and 1.8-6.1, and the intra- and inter-assay CV\% of the ghrelin kit was 0.90-1.91 and 5.18-7.81.

\subsection{Intervention Oils}

The intervention oils used in this study were extra virgin olive oil (Piccardo \& Savoré, Chiusavecchia, Italy) and free fatty acids of Siberian pine nut oil (Siberian Pine Nut Oil, Port Charlotte, FL 33952, USA). The later which fatty acid composition was analyzed and can be reviewed in a previous study by our group [1]. Hydrolysis of the pine nut oil was conducted as previously described [17]. Both oils were manually encapsulated in white semitransparent DRcaps ${ }^{\mathrm{TM}}$ from Capsugel ${ }^{\circledR}$ (size 00 batch: 5332311). These are resistant to acid and made of hydroxypropyl methylcellulose with delayed release properties. According to the manufacture, release is postponed approximately $45 \mathrm{~min}$ when using dry substance filling [26]. In a previous study, we conducted our own in vitro evaluation of the release profile of capsules filled with PNO-FFA and olive oil, and found an immediate but slow release of capsule content over $240 \mathrm{~min}$ [17]. Both intervention doses were $6 \mathrm{~g}$ of oil in total, which amounted to ten capsules (corresponding to $225 \mathrm{~kJ}$ ). Intervention doses were selected based on several factors, (1) the amount should be fit for long term studies and therefore realistic and safe to consume over a longer period of time, and potentially later as a marketable product, (2) hydrolyzed pine nut oil has not previously been consumed in larger doses, therefore due to safety concerns we used small doses and (3) hydrolyzed pine nut oil has been investigated in doses of 2-3 g in earlier studies, and shown to have effects on appetite regulating factors including gut hormone secretion and subjective appetite $[27,28]$. Subjects were blinded to oil type; however, a slight color difference was apparent for the individual oil types on the PNO-OO treatment. Oil capsules were kept at a temperature of $5{ }^{\circ} \mathrm{C}$ and stored $<3$ months.

\subsection{Outcomes and Statistics}

Treatment outcomes on glucose, insulin, C-peptide, GLP-1, GIP, ghrelin and FFA were evaluated and compared by calculating the total area under the curve (AUC) during the full six hours of testing $\left(\mathrm{AUC}_{\text {total }}\right)$ and for two hour segments $\left(\mathrm{AUC}_{0-120}, \mathrm{AUC}_{120-240}\right.$, and 
$\left.\mathrm{AUC}_{240-360}\right)$. Similarly, VAS treatment responses on appetite were calculated as AUCs for the full period of testing and two-hour segments. All AUCs were calculated by the use of the trapezoidal rule [29]. VAS treatment responses on gastrointestinal tolerability were assessed as changes during the OGTTs and treatment differences at 8 PM on the same evening after the OGTTs and at 8 AM the morning after the OGTTs.

Mixed model linear regression was used to compare AUCs of blood parameters and appetite. Model fit was assessed by qq-plots of the residuals and plots of residual versus fitted values. If these were unfit, data were transformed by the use of natural logarithm. We experienced a few missing data points on GIP (6/834), several on ghrelin (21/834) and a few on appetite $(5 / 2025)$. For GIP and ghrelin, in cases with only one missing data point from $30 \mathrm{~min}$ to $300 \mathrm{~min}$ (GIP 2 cases, ghrelin 7 cases), these were omitted in the calculation of the AUC, corresponding to imputing the mean value of results from the adjacent data points. For ghrelin, three subjects missed three or more consecutive results between 30 and $240 \mathrm{~min}$ of the test in one out of the three experimental arms. These were excluded prior to analysis, which meant that the PNO-FFA treatment was reduced to seven subjects and PNO-OO to six subjects. Moreover, data points missing at $360 \mathrm{~min}$ (GIP 3 cases, ghrelin 2 cases) were missing during analysis and model estimations were used in these cases. Changes in gastrointestinal symptoms were compared by the use of the non-parametric Wilcoxon signed rank test and adjusted using the Bonferroni-holm method. Significance was set to $p<0.05$.

\section{Results}

\subsection{Glucose, Insulin and C-Peptide}

There were no significant treatment differences in any of the calculated AUCs of glucose, insulin or C-peptide. Plasma glucose $\mathrm{AUC}_{120-240}$ tended to be slightly increased $(\sim 11 \%)$ after PNO-FFA versus no oil treatment $(p=0.098)$. OGTT response curves and corresponding AUCs are presented in Figure 1.

\subsection{Hormone Secretion of GLP-1, GIP and Ghrelin}

GLP-1 AUC $C_{\text {total }}$ and all two-hour AUC segments were increased ( 30-50\%) after PNO-FFA treatment compared to no oil $(p<0.01)$ (Figure 2A,B). Moreover, PNO-FFA also increased GLP-1 AUC $\mathrm{Atatal}_{\text {and }} \mathrm{AUC}_{120-240}(\sim 6-14 \%)$, in comparison to PNO-OO $(p<0.01)$, whereas, GLP-1 $\mathrm{AUC}_{0-120}$ and $\mathrm{AUC}_{240-360}$ only tended to be increased ( 14-21\%) after PNO-FFA versus PNO-OO $(p<0.1)$ (Figure 2A,B). PNO-FFA and PNO-OO increased GIP AUC $240-360(\sim 85-107 \%)$ compared to no oil $(p<0.01)$, and GIP AUC total $_{2}$ tended to be increased $(\sim 8 \%)$ after PNO-OO versus no oil $(p=0.066)$ (Figure $2 \mathrm{C}, \mathrm{D})$. None of the calculated ghrelin AUCs differed between treatments (Figure 2E,F).

\subsection{FFA}

PNO-FFA and PNO-OO reduced plasma FFA AUC $\mathrm{Atal}_{\text {tal }}$ compared to no oil $(p<0.01)$, and this was explained by reductions in the two last 2-h periods, $\mathrm{AUC}_{120-240}$ and $\mathrm{AUC}_{240-360}$ (all $p<0.01$ ) (Supplementary Figure S2). There were no differences in plasma FFA between PNO-FFA and PNO-OO. 



Figure 1. Glucose (A,B), insulin (C,D) and C-peptide (E,F) levels during a 6-h OGTT and their corresponding area under the curve calculated for 0-120, 120-240, 240-360 and 0-360 min in response to glucose alone (no oil, black bars and black circles), $6 \mathrm{~g}$ hydrolyzed pine nut oil (PNO-FFA, white bars and white squares) or $3 \mathrm{~g}$ hydrolyzed pine nut oil and $3 \mathrm{~g}$ olive oil (PNO-OO, gray bars and gray diamonds). Data are mean \pm SEM. 

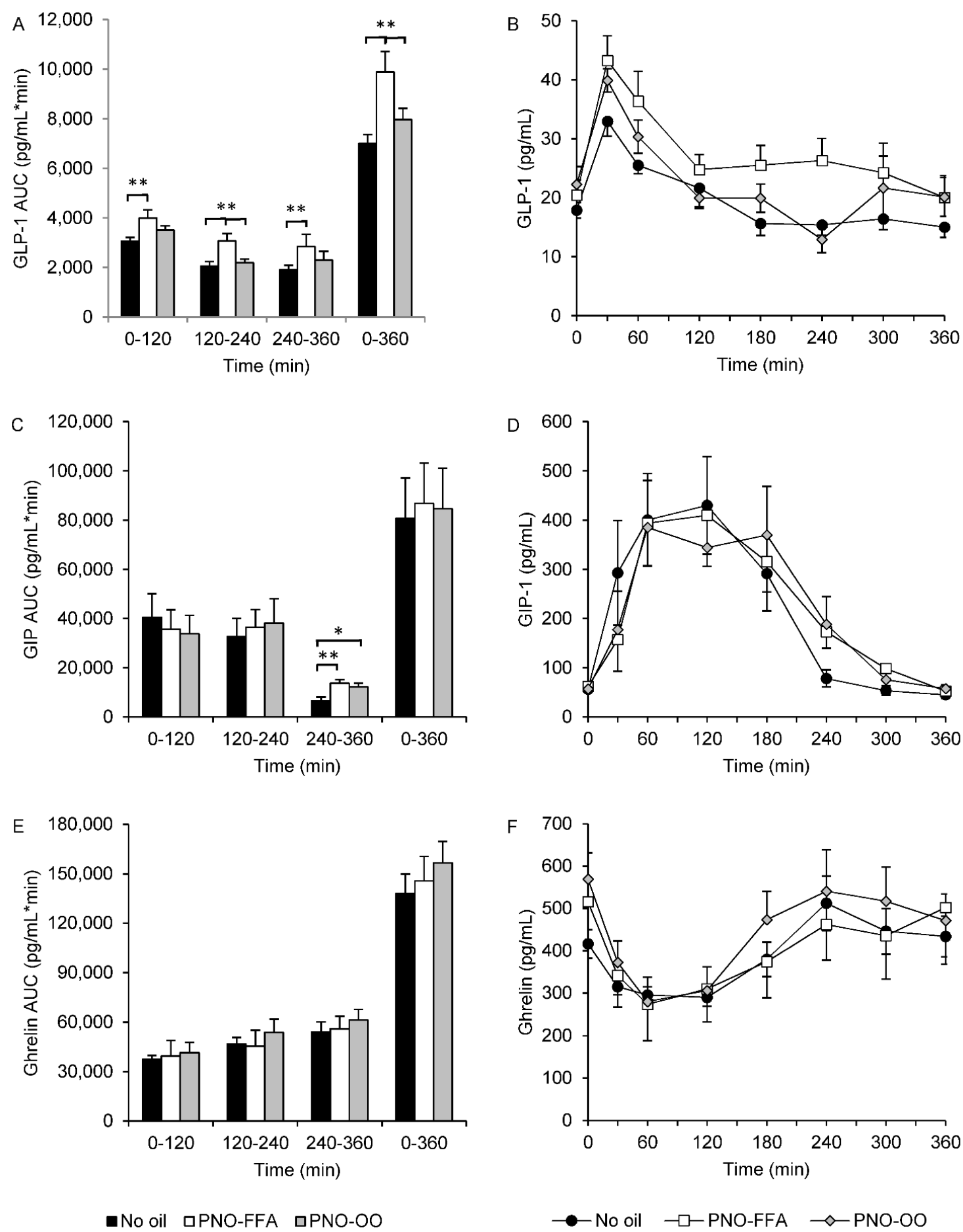

Figure 2. GLP-1 (A,B), GIP (C,D) and ghrelin (E,F) levels during a 6-h OGTT and their corresponding area under the curve calculated for 0-120, 120-240, 240-360 and 0-360 $\mathrm{min}$ in response to glucose alone (no oil, black bars and black circles), $6 \mathrm{~g}$ hydrolyzed pine nut oil (PNO-FFA, white bars and white squares) or $3 \mathrm{~g}$ hydrolyzed pine nut oil and $3 \mathrm{~g}$ olive oil (PNO-OO, gray bars and gray diamonds). Data are mean \pm SEM. ${ }^{*} p<0.05,{ }^{* *} p<0.01$.

\subsection{Insulin Sensitivity and Beta-Cell Function}

There were no treatment differences in the calculated OGTT-derived indices of insulin sensitivity. Similarly, indices of beta-cell function showed no significant differences between treatments (Table 2). 
Table 2. OGTT based indices on insulin sensitivity and beta-cell function.

\begin{tabular}{cccc}
\hline & No Oil & PNO-FFA & PNO-OO \\
\hline Insulin sensitivity & & & \\
BIGTT $_{\mathrm{Si}}$ & $3.7 \pm 0.9$ & $4.0 \pm 1.1$ & $4.0 \pm 0.8$ \\
MATSUDA $_{\text {Beta-cell function }}$ & $3.7 \pm 0.8$ & $4.0 \pm 1.0$ & $3.5 \pm 0.7$ \\
BIGTT $_{\text {AIR }}$ & $4362 \pm 641$ & $4807 \pm 1023$ & $4847 \pm 1109$ \\
CIR $_{30 \text { min }}$ & $1791 \pm 235$ & $2026 \pm 336$ & $1760 \pm 286$ \\
\hline
\end{tabular}

Values are mean \pm SEM.

\subsection{Subjective Appetite}

Satiety $\mathrm{AUC}_{\text {total }}$ increased after PNO-FFA and PNO-OO versus no oil ( $25-28 \%$; $p \leq 0.01$ for all). None of the two-hour satiety segments differed between treatments. Hunger $\mathrm{AUC}_{\text {total, }}, \mathrm{AUC}_{-30-120}$ and $\mathrm{AUC}_{240-360}$ decreased after PNO-FFA versus no oil $(\sim 6-18 \% ; p \leq 0.031$ for all). A decrease in hunger was also observed after PNO-OO versus no oil, though only for $\mathrm{AUC}_{\text {total }}$ and $\mathrm{AUC}_{240-360}(\sim 6 \%$ for both; $p<0.05$ for all). Fullness $\mathrm{AUC}_{\text {total, }} \mathrm{AUC}_{120-240}$ and $\mathrm{AUC}_{240-360}$ increased after PNO-OO versus no oil ( $24-47 \%$; $p \leq 0.045$ for all). An increase in fullness after PNO-FFA versus no oil was only observed for $\mathrm{AUC}_{240-360}(\sim 46 \% ; p=0.048)$. Prospective food consumption was reduced after both PNO-FFA and PNO-OO for $\mathrm{AUC}_{\text {total }}$ and all two-hour segments $(\sim 6-18 \% ; p \leq 0.045$ for all). Finally, overall well-being $\mathrm{AUC}_{120-240}$ was reduced after PNO-FFA versus no oil ( $\sim 12 \%$; $p=0.005)$. The two oil treatments did not differ in any of the assessed appetite symptoms. See Table 3, for all AUC appetite responses.

Table 3. Effects of PNO-FFA and PNO-OO on AUC appetite responses.

\begin{tabular}{|c|c|c|c|}
\hline & No Oil & PNO-FFA & PNO-OO \\
\hline \multicolumn{4}{|l|}{ Satiety } \\
\hline AUC-30-120 min & $5261 \pm 303^{1}$ & $6183 \pm 449$ & $5917 \pm 622$ \\
\hline $\mathrm{AUC}_{120-240 \mathrm{~min}}$ & $2733 \pm 359$ & $3220 \pm 285$ & $3785 \pm 511$ \\
\hline $\mathrm{AUC}_{240-360 \mathrm{~min}}$ & $1527 \pm 318$ & $2297 \pm 358$ & $2260 \pm 449$ \\
\hline $\begin{array}{c}\mathrm{AUC}_{\text {total }} \\
\text { Hunger }\end{array}$ & $9364 \pm 789^{1}$ & $11,700 \pm 822 *$ & $11,962 \pm 1390$ * \\
\hline $\mathrm{AUC}_{-30-120 \mathrm{~min}}$ & $9087 \pm 137^{1}$ & $7448 \pm 509 *$ & $8586 \pm 495$ \\
\hline $\mathrm{AUC}_{120-240 \mathrm{~min}}$ & $8202 \pm 529$ & $8113 \pm 442$ & $8052 \pm 429$ \\
\hline $\mathrm{AUC}_{240-360 \mathrm{~min}}$ & $9690 \pm 474$ & $9123 \pm 435^{*}$ & $9157 \pm 477 *$ \\
\hline $\begin{array}{l}\text { AUC }_{\text {total }} \\
\text { Fullness }\end{array}$ & $27,380 \pm 878^{1}$ & $24,684 \pm 1027$ * & $25,794 \pm 1123$ * \\
\hline $\mathrm{AUC}_{-30-120 \mathrm{~min}}$ & $4784 \pm 654^{1}$ & $5487 \pm 787$ & $5343 \pm 874$ \\
\hline $\mathrm{AUC}_{120-240 \mathrm{~min}}$ & $2268 \pm 363$ & $2782 \pm 459$ & $3245 \pm 687 *$ \\
\hline $\mathrm{AUC}_{240-360 \mathrm{~min}}$ & $1317 \pm 279$ & $1920 \pm 376^{*}$ & $1930 \pm 521 *$ \\
\hline \multicolumn{4}{|l|}{$\begin{array}{l}\text { Prospective food } \\
\text { consumption }\end{array}$} \\
\hline $\mathrm{AUC}_{-30-120 \mathrm{~min}}$ & $9815 \pm 560^{1}$ & $8082 \pm 759 *$ & $8819 \pm 753$ * \\
\hline $\mathrm{AUC}_{120-240 \mathrm{~min}}$ & $9098 \pm 436$ & $8348 \pm 501 *$ & $8387 \pm 603 *$ \\
\hline $\mathrm{AUC}_{240-360 \mathrm{~min}}$ & $9797 \pm 496$ & $8993 \pm 630$ * & $9227 \pm 687 *$ \\
\hline $\mathrm{AUC}_{\text {total }}$ & $28,850 \pm 1442^{1}$ & $25,423 \pm 1658 *$ & $26,433 \pm 1847$ * \\
\hline \multicolumn{4}{|l|}{ Overall well-being } \\
\hline $\mathrm{AUC}_{-30-120 \mathrm{~min}}$ & $12,111 \pm 877^{1}$ & $10,256 \pm 1187$ & $11,184 \pm 1073$ \\
\hline $\mathrm{AUC}_{120-240 \mathrm{~min}}$ & $9150 \pm 676$ & $8095 \pm 953 *$ & $9022 \pm 769$ \\
\hline $\mathrm{AUC}_{240-360 \mathrm{~min}}$ & $8486 \pm 793$ & $8070 \pm 918$ & $9000 \pm 713$ \\
\hline $\mathrm{AUC}_{\text {total }}$ & $30,004 \pm 1993^{1}$ & $26,421 \pm 2981$ & $29,206 \pm 2496$ \\
\hline
\end{tabular}

VAS appetite responses calculated as area under the curve (AUC, $\mathrm{mm}^{*} \mathrm{~min}$ ). Values are mean \pm SEM. Superscript numerals indicate number of values missing completely at random. ${ }^{*} p<0.05$ versus no oil. 


\subsection{Gastrointestinal Tolerability}

The delta value of flatulence decreased during the OGTT after no oil versus PNO-OO treatment $(p=0.023)$ and, also a tendency of a decrease after no oil versus PNO-FFA $(p=0.07)$ was observed. No treatment differences in delta values of bloating, diarrhea or constipation during the OGTT were observed and neither were there any treatment differences in gastrointestinal symptoms at 8PM or 8AM after the OGTTs. Outcomes on gastrointestinal tolerability are presented in Supplementary Table S1.

\section{Discussion}

Our major aim was to investigate whether combined intake of selected dietary lipids, could act in synergy to enhance GLP-1 secretion and consequently reduce appetite and improve insulin secretion and glucose tolerance in humans in vivo. This was done by testing the effects of delayed-release hydrolyzed pine nut oil, containing pinolenic acid, a potent dual FFA1/FFA4 agonist, in combination with olive oil, liberating the GPR119 agonist $2 \mathrm{OG}$ upon digestion, versus intake of hydrolyzed pine nut oil alone or no oil intake prior to an OGTT.

We detected no enhanced effect of combining hydrolyzed pine nut oil and olive oil on GLP-1 secretion when delivered to the small intestine by delayed release capsules. This is in contrast to a recent preclinical study showing a strong synergistic effect of activating both FFA1 and GPR119 in mouse colonic crypt cells on GLP-1 secretion [7]. One explanation may be the doses of pine nut oil and olive oil in our study, which may have been too small to activate FFA1 and GPR119 in vivo in humans. It has been found that $19 \mathrm{~g}$ of olive oil, which is converted to $7.7 \mathrm{~g}$ of $2 \mathrm{OG}$ upon complete digestion, increases postprandial GLP-1 secretion in humans versus no oil intake [18]. Our PNO-OO treatment containing $3 \mathrm{~g}$ of olive oil would contribute $1.2 \mathrm{~g}$ of $2 \mathrm{OG}$ upon digestion, only a 6th of the amount used by Mandøe et al. [18]. However, it has been demonstrated in humans that a relatively small dose of $2 \mathrm{~g}$ of $2 \mathrm{OG}$ given as a bolus through a nasojejunal tube was sufficient to increase GLP-1 levels from 0-25 min after administration [19]. This indicates that the administration of 2OG directly to the more distal part (jejunum) of the small intestine will be more efficient in activating GPR119 and hence increase GLP-1 secretion. In our study, pine nut oil and olive oil were delivered to the small intestine in delayed-release capsules, which has been shown to give a prolonged release profile in vitro [17]. Therefore, using this administration method the release site is much more uncertain as compared to both the preclinical study using a direct method by stimulating a specific cell line of colonic crypt cells [6] as well as the clinical study giving 2OG through a nasojejunal tube [19]. An early release would lead to hydrolysis in the upper intestinal tract and absorption before the receptor populations in the lower intestinal tract is reached, whereas a late release might lead to incomplete enzymatic hydrolysis and a lower amount of 2OG. It is therefore possible, that a less specific gastrointestinal release site together with a smaller dose diminished a potential stimulatory effect of 2OG in our study. Future studies addressing this issue by using a formulation that ensures release in the lower gut and possibly larger doses, preferably using purified 2OG to eliminate the dependence on enzymatic hydrolysis, are needed.

Importantly, we found a pronounced effect of $6 \mathrm{~g}$ PNO-FFA intake alone on GLP-1 AUC compared to both PNO-OO and no oil intake from 0-360 min of the OGTT. This effect was significant for all $2 \mathrm{~h}$ periods during the OGTT when compared to no oil, but only from 120-240 min compared to PNO-OO. These results are in line with our previous studies, which mainly demonstrated an increase in GLP-1 secretion 120-240 min after intake of PNO-FFA versus no oil [17]. Thus, the effect of delayed-release hydrolyzed pine nut oil on GLP-1 secretion seems to be a consistent and reproducible outcome. Interestingly, in the present study GLP-1 secretion was significantly increased already from 0-120 min of the OGTT. A similar pattern was seen in our previous study [17], however, these differences were less pronounced and did not reach significance. Ingestion of high doses of olive oil $(30 \mathrm{~mL})$ or oleic acid $(0.88 \mathrm{~g} / \mathrm{kg}$ body weight) have been reported to increase circulating GLP-1 levels suggesting a general effect of oil intake on GLP-1 secretion [30,31]. However, 
while intake of $6 \mathrm{~g}$ oil either as PNO-FFA or PNO-OO caused a similar reduction in plasma FFA from 120-360 min compared to no oil, only the double dose of hydrolyzed pine nut oil significantly increased GLP-1 levels. This support a specific effect of hydrolyzed pine nut oil on GLP-1 rather than a general effect of oils, at least at lower doses. Noteworthy, in this study we prolonged the acute OGTT test up to $360 \mathrm{~min}$ vs. $240 \mathrm{~min}$ in our previous study, as the previous results on GLP-1 indicated enhanced secretion after both 3 and $6 \mathrm{~g}$ PNO-FFA beyond the $240 \mathrm{~min}$ of testing. We were able to confirm this assumption, though the enhanced secretion slowly attenuates from 240-360 min.

Glucose tolerance was unaffected by the treatments in the present study. Thus, neither glucose, insulin, nor C-peptide responses showed any significant treatment differences. This is to some extent in contrast to our previous studies, in which we observed small reductions in glucose and insulin responses from 0-120 min of the OGTT after 3 and $6 \mathrm{~g}$ PNO-FFA intake versus no oil [17]. Overall, our results indicate less clear effects of PNO-FFA on glucose tolerance, and no enhancement when combining hydrolyzed pine nut oil with olive oil. Moreover, the present study shows that while treatment with PNO-FFA enhances GLP-1 secretion throughout the test, it does not convincingly increase insulin secretion or reduce glucose levels. The question is whether such effects can be expected in subjects with a normal glucose tolerance. Therefore, it would be important to test the acute effects of PNO-FFA in prediabetic or type 2 diabetic subjects in future studies, before potential beneficial effects on glucose and insulin secretion can be ruled out.

In this study, we observed no significant differences in ghrelin levels between any of the treatments. This is consistent with a previous study showing similar ghrelin responses after intake of $3 \mathrm{~g}$ doses of pine nut oil, pine nut oil free fatty acids or olive oil [28]. Thus, intake of these oils seems to affect ghrelin levels to a similar degree when given separately. However, in the present study, there were no differences between intakes of oils versus no oil, which is in contrast to what we have observed previously [17]. It is however consistent with the ghrelin receptor being primarily expressed in the upper gastrointestinal tract [32], whereas the delayed-release formulation targets the lower intestines. In comparison to our earlier studies, we experienced a substantial number of missing data on ghrelin levels as described above. This meant that the PNO-FFA treatment arm was reduced to seven subjects and the PNO-OO arm to six subjects, which may have reduced the power of the study and compromised the validity of the ghrelin results.

GIP secretion was unaffected by oil type from $0-240 \mathrm{~min}$ in this study. Across our own studies, we have not been able to reproduce consistent findings on GIP secretion. Previously we have reported that PNO-FFA $3 \mathrm{~g}$ versus no oil reduced GIP secretion from 0-120 min. and increased secretion from 120-240 min. But this result was not reproduced in our dose response study, and neither in this study. Moreover, we observed an increase in GIP secretion from 240-360 min after both oil types in this study, suggesting no distinct effect of oil type on GIP secretion. It could be speculated, that these inconsistent findings may be driven by the smaller abundancy of GIP secreting K-cells in the distal intestine (release site), combined with the less specific release profile of the capsules as described previously leading to different results. Therefore, further studies are necessary. Satiety and prospective food consumption were affected similarly with an appetite reducing effect after PNO-FFA and PNO-OO compared to no oil. Hunger was also reduced after both oil treatment versus no oil. PNO-FFA seemed to have the most pronounced effect with several two-hour segments being significant. This was opposite for fullness, where both oil treatments reduced fullness from 4-6 h versus no oil, while several AUCs were reduced after PNO-OO suggesting a more pronounced effect. Only PNO-FFA seemed to reduce overall well-being versus no oil from $2-4 \mathrm{~h}$ of the test. Despite a few differences, the overall picture was a similar appetite reducing effect of the two oil treatments. Despite an increase in the appetite suppressive hormone GLP-1 only after PNO-FFA, this did not significantly reduce the subjective appetite further in comparison to PNO-OO. It has been suggested that free fatty acids of pine nut oil, corresponding to our PNO-FFA treatment, is able to decrease appetite by reducing prospective food consumption in comparison to intake of 
olive oil alone [28]. However, we did not observe significant differences between the oil treatments, suggesting similar appetite reducing effects independent of oil type. Moreover, we did not include a pure olive oil arm in our study, and, therefore it is possible that the $3 \mathrm{~g}$ hydrolyzed pine nut oil in the PNO-OO treatment was sufficient to reduce appetite to the same extent as the $6 \mathrm{~g}$ PNO-FFA alone. This is consistent with the findings in our previous dose-response study, where we observed similar reductions in subjective appetite after intake of 3 and $6 \mathrm{~g}$ PNO-FFA, respectively [17]. Finally, an important limitation in our study is the lack of a blinded placebo treatment. The participants knew whether they received oil or not, which may have influenced the VAS results on appetite measures.

Gastrointestinal tolerability of the oil capsules was good. A reduction in flatulence during the OGTT after no oil compared to PNO-OO was observed. It is likely that this change reflects an increased level already prior to the OGTT, and therefore does not reflect an actual ameliorating effect of glucose intake compared to the oil treatments.

The major limitation of our study is the unblinded design in respect to no oil versus the two oil intake days. Moreover, the relatively small sample size made data susceptible for reduced validity when missing data occurred.

\section{Conclusions}

In conclusion, we did not observe an enhanced effect on GLP-1 secretion, glucose tolerance or appetite when combing oral intake of hydrolyzed pine nut oil (containing $\sim 20 \%$ pinolenic acid) with olive oil (releasing $2 \mathrm{OG}$ ) to potentially stimulate FFA1, FFA4 and GPR119 in the small intestine. Importantly, however, we could reproduce that hydrolyzed pine nut oil delivered to the small intestine by delayed-release capsules augments GLP-1 secretion. As GLP-1 is a known important gut hormone regulating glucose metabolism, subjective appetite and body weight it is relevant to further investigate whether treatment with hydrolyzed pine nut oil in delayed release capsules may be a future dietary supplement with potential beneficial effects on these outcomes in well-powered acute and long-term studies, and preferably in subject with prediabetes or overt type 2 diabetes.

Supplementary Materials: The following are available online at https:/ /www.mdpi.com/article/10.3 390/nu13103407/s1, Figure S1: Study design, Figure S2: Free fatty acids (FFA) levels during a 6-h OGTT, Table S1: Changes in gastrointestinal tolerability during the OGTT, at 8PM after the OGTT and at $8 \mathrm{AM}$ after the OGTT.

Author Contributions: Conceptualization, K.H., T.U. and K.V.S.; methodology, K.H., T.U., A.B.-B., M.H.K., J.H.E. and K.V.S.; validation, K.H.; formal analysis, K.V.S.; investigation, K.V.S.; data curation, K.V.S.; writing—original draft preparation, K.V.S.; writing—review and editing, T.U., A.B.-B., M.H.K., J.H.E., K.V.S. and K.H.; visualization, K.V.S.; supervision, K.H. and T.U.; project administration, K.V.S.; funding acquisition, T.U., K.H. and K.V.S. All authors have read and agreed to the published version of the manuscript.

Funding: This research was funded by the Danish Research Council for Strategic Research (grant 11-116196) and Innovation Fund Denmark (grant 0603-00452B). In addition, Odense University Hospital, the Region of Southern Denmark, the University of Southern Denmark (SDU2020) and the Ingemann O. Bucks fund supplied financial support.

Institutional Review Board Statement: The study was conducted according to the guidelines of the Declaration of Helsinki and approved by the Regional Committees on Health Research Ethics for Southern Denmark (S-20150060).

Informed Consent Statement: Informed consent was obtained from all subjects involved in the study.

Data Availability Statement: The data presented in this study can be requested from the corresponding author.

Acknowledgments: We thank Lone Hansen and Charlotte Bøtchiær Olsen for technical assistance. The study was a part of the FFARMED project lead by Trond Ulven (http:/ /ffarmed.dk/, 27 September 2021). We thank the entire project group for valuable inputs and discussions throughout the process.

Conflicts of Interest: The authors declare no conflict of interest. 


\section{References}

1. Christiansen, E.; Watterson, K.R.; Stocker, C.J.; Sokol, E.; Jenkins, L.; Simon, K.; Grundmann, M.; Petersen, R.K.; Wargent, E.; Hudson, B.; et al. Activity of dietary fatty acids on FFA1 and FFA4 and characterisation of pinolenic acid as a dual FFA1/FFA4 agonist with potential effect against metabolic diseases. Br. J. Nutr. 2015, 113, 1677-1688. [CrossRef]

2. Yang, J.W.; Kim, H.S.; Choi, Y.; Kim, Y.; Kang, K.W. Therapeutic application ofGPR119ligands in metabolic disorders. Diabetes Obes. Metab. 2017, 20, 257-269. [CrossRef] [PubMed]

3. Hansen, H.S.; Rosenkilde, M.M.; Holst, J.J.; Schwartz, T.W. GPR119 as a fat sensor. Trends Pharmacol. Sci. 2012, 33, 374-381. [CrossRef] [PubMed]

4. Edfalk, S.; Steneberg, P.; Edlund, H. Gpr40 is expressed in enteroendocrine cells and mediates free fatty acid stimulation of incretin secretion. Diabetes 2008, 57, 2280-2287. [CrossRef] [PubMed]

5. Hirasawa, A.; Tsumaya, K.; Awaji, T.; Katsuma, S.; Adachi, T.; Yamada, M.; Sugimoto, Y.; Miyazaki, S.; Tsujimoto, G. Free fatty acids regulate gut incretin glucagon-like peptide-1 secretion through GPR120. Nat. Med. 2004, 11, 90-94. [CrossRef]

6. Iwasaki, K.; Harada, N.; Sasaki, K.; Yamane, S.; Iida, K.; Suzuki, K.; Hamasaki, A.; Nasteska, D.; Shibue, K.; Joo, E.; et al. Free Fatty Acid Receptor GPR120 Is Highly Expressed in Enteroendocrine K Cells of the Upper Small Intestine and Has a Critical Role in GIP Secretion After Fat Ingestion. Endocrinology 2015, 156, 837-846. [CrossRef] [PubMed]

7. $\quad$ Ekberg, J.H.; Hauge, M.; Kristensen, L.V.; Madsen, A.N.; Engelstoft, M.S.; Husted, A.S.; Sichlau, R.; Egerod, K.L.; Timshel, P.; Kowalski, T.J.; et al. GPR119, a Major Enteroendocrine Sensor of Dietary Triglyceride Metabolites Coacting in Synergy with FFA1 (GPR40). Endocrinology 2016, 157, 4561-4569. [CrossRef] [PubMed]

8. Nauck, M.A.; Meier, J.J. Incretin hormones: Their role in health and disease. Diabetes Obes. Metab. 2018,20 (Suppl. 1), 5-21. [CrossRef]

9. Andersen, A.; Lund, A.; Knop, F.K.; Vilsbøll, T. Glucagon-like peptide 1 in health and disease. Nat. Rev. Endocrinol. 2018, 14, 390-403. [CrossRef]

10. Itoh, Y.; Kawamata, Y.; Harada, M.; Kobayashi, M.; Fujii, R.; Fukusumi, S.; Ogi, K.; Hosoya, M.; Tanaka, Y.; Uejima, H.; et al. Free fatty acids regulate insulin secretion from pancreatic beta cells through GPR40. Nature 2003, 422, 173-176. [CrossRef]

11. Chu, Z.L.; Jones, R.M.; He, H.; Carroll, C.; Gutierrez, V.; Lucman, A.; Moloney, M.; Gao, H.; Mondala, H.; Bagnol, D.; et al. A role for beta-cell-expressed $\mathrm{G}$ protein-coupled receptor 119 in glycemic control by enhancing glucose-dependent insulin release. Endocrinology 2007, 148, 2601-2609. [CrossRef]

12. Kaku, K.; Enya, K.; Nakaya, R.; Ohira, T.; Matsuno, R. Efficacy and safety of fasiglifam (TAK-875), a G protein-coupled receptor 40 agonist, in Japanese patients with type 2 diabetes inadequately controlled by diet and exercise: A randomized, double-blind, placebo-controlled, phase III trial. Diabetes Obes. Metab. 2015, 17, 675-681. [CrossRef]

13. Engelstoft, M.S.; Park, W.-M.; Sakata, I.; Kristensen, L.V.; Husted, A.S.; Osborne-Lawrence, S.; Piper, P.K.; Walker, A.K.; Pedersen, M.H.; Nøhr, M.K.; et al. Seven transmembrane G protein-coupled receptor repertoire of gastric ghrelin cells. Mol. Metab. 2013, 2, 376-392. [CrossRef]

14. Mihalache, L.; Gherasim, A.; Niță, O.; Ungureanu, M.C.; Pădureanu, S.S.; Gavril, R.S.; Arhire, L.I. Effects of ghrelin in energy balance and body weight homeostasis. Hormones 2016, 15, 186-196. [CrossRef]

15. Oh, D.Y.; Talukdar, S.; Bae, E.J.; Imamura, T.; Morinaga, H.; Fan, W.; Li, P.; Lu, W.J.; Watkins, S.M.; Olefsky, J.M. GPR120 Is an Omega-3 Fatty Acid Receptor Mediating Potent Anti-inflammatory and Insulin-Sensitizing Effects. Cell 2010, 142, 687-698. [CrossRef] [PubMed]

16. Oh, D.Y.; Walenta, E.; Akiyama, T.E.; Lagakos, W.S.; Lackey, D.; Pessentheiner, A.; Sasik, R.; Hah, N.; Chi, T.J.; Cox, J.M.; et al. A Gpr120-selective agonist improves insulin resistance and chronic inflammation in obese mice. Nat. Med. 2014, 20, 942-947. [CrossRef] [PubMed]

17. Sørensen, K.V.; Korfitzen, S.S.; Kaspersen, M.H.; Ulven, E.R.; Ekberg, J.H.; Bauer-Brandl, A.; Ulven, T.; Højlund, K. Acute effects of delayed-release hydrolyzed pine nut oil on glucose tolerance, incretins, ghrelin and appetite in healthy humans. Clin. Nutr. 2021, 40, 2169-2179. [CrossRef] [PubMed]

18. Mandøe, M.J.; Hansen, K.B.; Windeløv, J.A.; Knop, F.K.; Rehfeld, J.F.; Rosenkilde, M.M.; Holst, J.J.; Hansen, H.S. Comparing olive oil and C4-dietary oil, a prodrug for the GPR119 agonist, 2-oleoyl glycerol, less energy intake of the latter is needed to stimulate incretin hormone secretion in overweight subjects with type 2 diabetes. Nutr. Diabetes 2018, 8, 2. [CrossRef]

19. Hansen, K.B.; Rosenkilde, M.M.; Knop, F.K.; Wellner, N.; Diep, T.A.; Rehfeld, J.F.; Andersen, U.B.; Holst, J.J.; Hansen, H.S. 2-Oleoyl Glycerol Is a GPR119 Agonist and Signals GLP-1 Release in Humans. J. Clin. Endocrinol. Metab. 2011, 96, E1409-E1417. [CrossRef]

20. Mandoe, M.J.; Hansen, K.B.; Hartmann, B.; Rehfeld, J.F.; Holst, J.J.; Hansen, H.S. The 2-monoacylglycerol moiety of dietary fat appears to be responsible for the fat-induced release of GLP-1 in humans. Am. J. Clin. Nutr. 2015, 102, 548-555. [CrossRef]

21. Flint, A.; Raben, A.; Blundell, J.; Astrup, A. Reproducibility, power and validity of visual analogue scales in assessment of appetite sensations in single test meal studies. Int. J. Obes. 2000, 24, 38-48. [CrossRef] [PubMed]

22. Bengtsson, M.; Persson, J.; Sjölund, K.; Ohlsson, B. Further Validation of the Visual Analogue Scale for Irritable Bowel Syndrome after Use in Clinical Practice. Gastroenterol. Nurs. 2013, 36, 188-198. [CrossRef] [PubMed]

23. Hansen, T.; Drivsholm, T.; Urhammer, S.A.; Palacios, R.T.; Vølund, A.; Borch-Johnsen, K.; Pedersen, O. The BIGTT test: A novel test for simultaneous measurement of pancreatic beta-cell function, insulin sensitivity, and glucose tolerance. Diabetes Care 2007, 30, 257-262. [CrossRef] [PubMed] 
24. Matsuda, M.; DeFronzo, R.A. Insulin sensitivity indices obtained from oral glucose tolerance testing: Comparison with the euglycemic insulin clamp. Diabetes Care 1999, 22, 1462-1470. [CrossRef] [PubMed]

25. Sluiter, W.J.; Erkelens, D.W.; Reitsma, W.D.; Doorenbos, H. Glucose tolerance and insulin release, a mathematical approach I. Assay of the beta-cell response after oral glucose loading. Diabetes 1976, 25, 241-244. [CrossRef]

26. ProductDescription_DRcaps ${ }^{\mathrm{TM}}$. A Matter of Timing. Ensure Optimal Delivery for Acid-Sensitive Products. 2016. Available online: https://s3.amazonaws.com/cpsl-web/kc/library/c1a-32029_DRCaps-A4_FIN.PDF (accessed on 22 January 2019).

27. Hughes, G.M.; Boyland, E.J.; Williams, N.J.; Mennen, L.; Scott, C.; Kirkham, T.C.; Harrold, J.A.; Keizer, H.G.; Halford, J.C. The effect of Korean pine nut oil (PinnoThin) on food intake, feeding behaviour and appetite: A double-blind placebo-controlled trial. Lipids Health Dis. 2008, 7, 6. [CrossRef]

28. Pasman, W.J.; Heimerikx, J.; Rubingh, C.M.; Berg, R.V.D.; O’Shea, M.; Gambelli, L.; Hendriks, H.F.; Einerhand, A.W.; Scott, C.; Keizer, H.G.; et al. The effect of Korean pine nut oil on in vitro CCK release, on appetite sensations and on gut hormones in post-menopausal overweight women. Lipids Health Dis. 2008, 7, 10. [CrossRef]

29. Curry, S.H.; Whelpton, R. Appendix 1. Mathematical Consepts and the Trapezoidal Method. In Introduction to Drug Disposition and Pharmacokinetics; John Wiley \& Sons, Ltd.: Chichester, UK, 2017; pp. 293-299.

30. Carr, R.D.; Larsen, M.O.; Winzell, M.S.; Jelic, K.; Lindgren, O.; Deacon, C.F.; Ahren, B. Incretin and islet hormonal responses to fat and protein ingestion in healthy men. Am. J. Physiol. Metab. 2008, 295, E779-E784. [CrossRef]

31. Gentilcore, D.; Chaikomin, R.; Jones, K.; Russo, A.; Feinle-Bisset, C.; Wishart, J.M.; Rayner, C.K.; Horowitz, M. Effects of Fat on Gastric Emptying of and the Glycemic, Insulin, and Incretin Responses to a Carbohydrate Meal in Type 2 Diabetes. J. Clin. Endocrinol. Metab. 2006, 91, 2062-2067. [CrossRef]

32. Engelstoft, M.; Schwartz, T. Opposite Regulation of Ghrelin and Glucagon-like Peptide-1 by Metabolite G-Protein-Coupled Receptors. Trends Endocrinol. Metab. 2016, 27, 665-675. [CrossRef] 